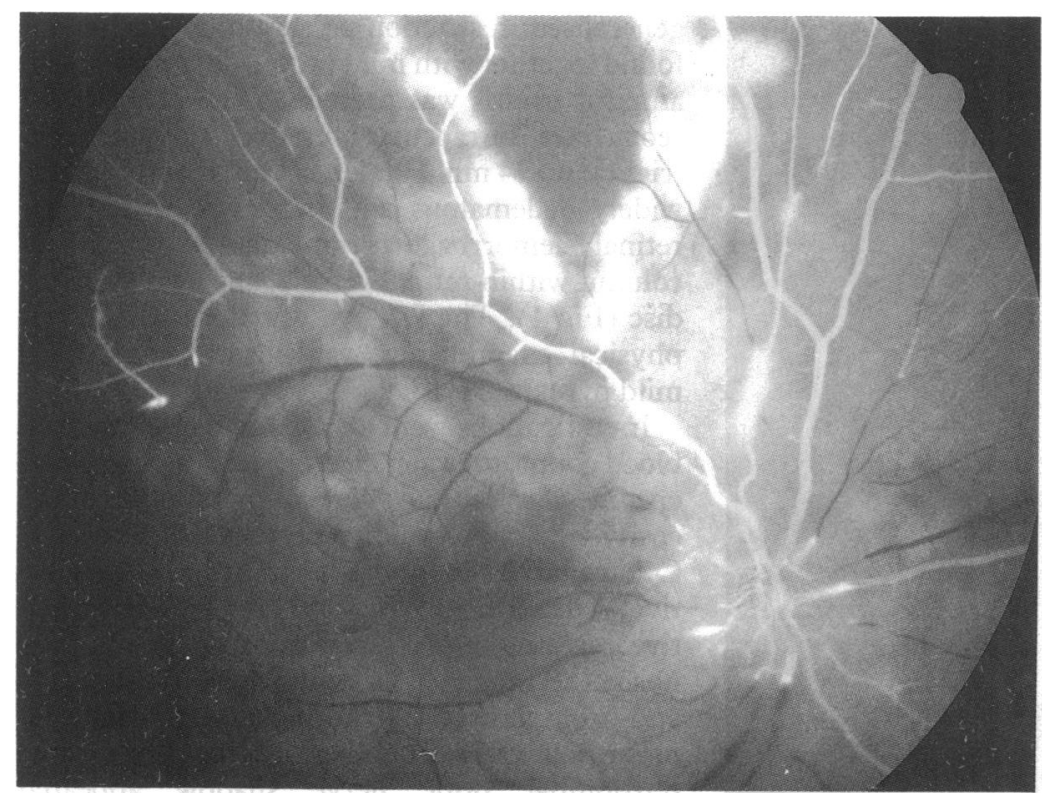

Figure 3 Posterior scleritis: fluorescein angiogram demonstrating multiple retinal arterial occlusions with vessel staining and segmental choroidal ischaemia.

retinal detachment were also found in one case by Cleary et $a l .{ }^{4}$ Review of the current literature failed to identify any cases where retinal vasculitis predominated or where choroidal infarction occurred. Optic disc swelling is a recognised feature of posterior scleritis. In this case with such extensive vascular involvement it is possible that direct infarction of the optic nerve head could have occurred; however, the clinical findings can be explained without the need to postulate such an event. Prospective studies of fluorescein angiography in posterior scleritis have not been performed. This is probably due to the rarity of the lesions and their polymorphic nature. No pathognomonic features have been described. The angiographic features described here, although striking, are not specific.

The photographs were taken by Mr Hugh Harris.

1 Calthorpe CM, Watson PG, McCartney ACE. Posterior scleritis: a clinical and histological survey. Eye 1988; 2: 26777.

2 Wilhelmus KR, Grierson I, Watson PG. Histopathologic and clinical associations of scleritis and glaucoma. Am $\mathcal{F}$ Ophthalmol 1981; 91: 697-705.

3 Bonin P. Cases reported at the Fan Club Meeting, Bonn, November 1984. Case 2. Acute posterior scleritis. Int Ophthalmol 1985; 8: 217-20

4 Cleary PE, Watson PG, McGill JI, Hamilton AM. Visual loss due to posterior segment disease in scleritis. Trans Ophthalmol Soc UK 1975; 95: 297-300.

\title{
Ocular albinism with unilateral sectorial pigmentation in the fundus
}

\author{
Takashi Shiono, Tsutomu Mutoh, Yasushi Chida, Makoto Tamai
}

\author{
Department of \\ Ophthalmology, Tohoku \\ University School of \\ Medicine, 1-1 Seiryo- \\ machi, Aoba-ku, Sendai \\ 980, Japan \\ T Shiono \\ T Mutoh \\ Y Chida \\ M Tamai \\ Correspondence to: \\ T Shiono, MD. \\ Accepted for publication \\ 24 November 1993
}

A diagnosis of ocular albinism ${ }^{12}$ is usually made in individuals with nystagmus, diminished visual acuity, reduced iris and fundus pigment, and foveal hypoplasia. Hypopigmentation of the hair and skin is not found in people with ocular albinism.

We describe a Japanese woman who had ocular albinism with good visual acuity in the left eye and no nystagmus. Her right eye showed low visual acuity. Interestingly, sectorial pigmentation, which reached in part to the macula, was seen only in her left fundus. No pigmentation was noted in the right fundus. To the best of our knowledge, no reports have been written previously about patients with this type of asymmetrical ocular albinism and good visual acuity.

\section{Case report}

A 60-year-old Japanese woman was referred to our clinic on 15 March 1991, for an ophthalmic examination after retinal changes were discovered during a routine examination of her fundus. She had mild diabetes mellitus, no ocular complaints, and no hearing loss. She had been aware of her hypopigmented irides since childhood. Her parents had brown irides. Their marriage was not consanguineous. The patient's three siblings and two daughters had brown irides. No one in her family was reported to have had nystagmus or other eye diseases including hypopigmentation.

Examination showed the patient to have dark brown hair, brown eyebrows, and brown eyelashes. Her skin was not albinotic. No spontaneous nystagmus or strabismus were detected. Her visual acuity was $20 / 40$ in her right eye, with a refractive correction of $-7 \cdot 75 \mathrm{D}$, and $20 / 20$ in her left eye, without correction; however, no stereopsis was found with use of a Randot stereotest. Slit-lamp examination of the anterior segment showed bluish-grey irides with a brown 
Figure 1 Marked hypopigmentation is noted in the right fundus. It is very difficult to locate the macula without pigmentation. Myopic conus is also seen.

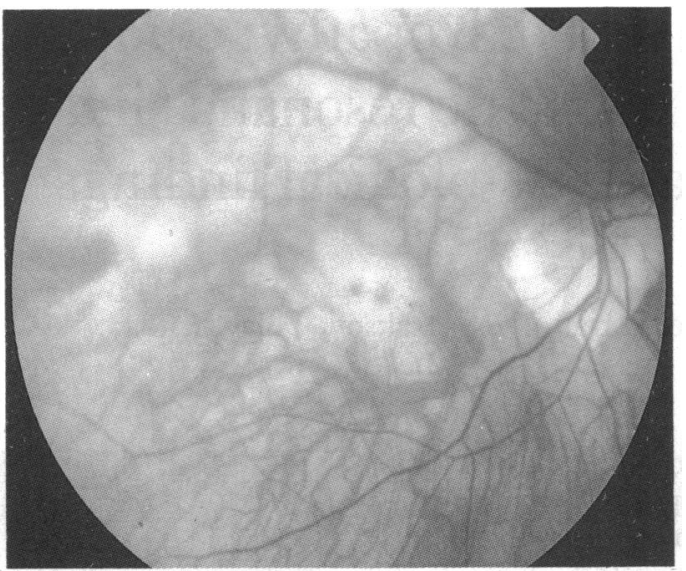

pupillary margin. Both lenses were clear. Fundus examination revealed no visible melanin pigment. Consequently, the choroidal vessels could be seen (Fig 1). However, a sectorial region of pigmented choroid was noticed temporally in the left fundus, which included part of the macula (Fig 2). Although it was difficult to locate the fovea in the albino fundus, both eyes seemed to have one. Fluorescein angiography of both eyes revealed a hypofluorescent area that corresponded to the macula, confirming the presence of xanthophyllic pigment. The pigmented area of the left eye also showed hypofluorescence. Single flash bright electroretinogram (ERG) with intense stimulus ( 25 joule bright white flash) was recorded after 30 minutes of dark adaptation. Thereafter, a $30 \mathrm{~Hz}$ red $(660 \mathrm{~nm})$ flicker ERG was recorded after 20 minutes of light adaptation. Results of these ERGs appeared normal.

\section{Comment}

Our female patient showed clinical signs of ocular albinism but none of oculocutaneous albinism. Her hair, eyebrows, and eyelashes were dark brown and her skin was not albinotic, although her irides and her fundi showed depigmentation. She had no stereopsis. Hypopigmentation of the eye in female patients without systemic diseases has been found in patients with autosomal recessive ocular albinism ${ }^{12}$ and in female carriers for $\mathrm{X}$ linked ocular albinism. ${ }^{3}$ The family history of our patient could rule out her being a female carrier for $\mathrm{X}$ linked ocular albinism. The absence of ocular abnormalities in the patient's parents, siblings, and children suggests that the disorder is an autosomal recessively inherited trait or a manifestation of sporadic ocular albinism. Japanese people always have brown irides. Blue or grey irides are exhibited only in Japanese people who have ocular abnormalities such as albinism, Waardenburg's syndrome, or heterochromic uveitis.

Autosomal dominant oculocutaneous albin-

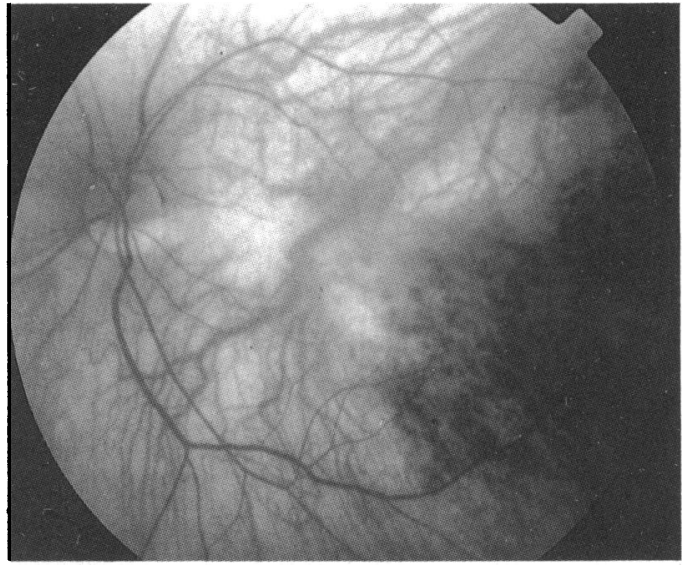

Figure 2 Marked hypopigmentation is noted in the left eye. Sectorial pigmentation of the choroid and retinal pigment epithelium is apparent in the temporal area of the fundus, including part of the macula.

oidism ${ }^{4}$ could also be differentiated from the condition in our patient. Although patients with oculocutaneous albinoidism have good visual acuity and no nystagmus, they had an albinotic complexion and eyes with normal foveas. Also, inheritance is autosomal dominant. Our patient had no stereopsis, albinotic skin, or albinotic hair. She had low visual acuity in the right eye, which showed no pigmentation. Good visual acuity was noted only in the left eye where pigmentation reached in part to the macula. The good visual acuity in the right eye, therefore, may be attributed to the pigmentation in the macula, suggesting that the condition in our patient appeared to be ocular albinism, not albinoidism. It may be possible, however, that the decreased visual acuity in the right eye resulted from anisometropic amblyopia due to myopia in the right eye. Conversely, the myopia in the right eye might have been a secondary change such as form deprived myopia, ${ }^{5}$ caused by unilateral congenital low visual acuity resulting from the depigmentation in the macula. Therefore, the most likely diagnosis of our patient's condition seemed to be an autosomal recessive or sporadic ocular albinism with unilateral sectorial pigmentation.

This study was supported in part by grant in aid for scientific research from the Ministry of Education, Science and Culture, Japan.

1 Scialfa A. Ocular albinism in a female. Am $\mathcal{F}$ Ophthalmol 1972 73: 943-8.

2 O'Donnell FE, King RA, Green WR, Witkop CJ. Autosoma recessively inherited ocular albinism. Arch Ophthamol 1978; 96: $1621-5$.

3 Jaeger C, Jay B. X-linked ocular albinism. A family containing a manifesting heterozygote, and an affected male married to female with autosomal recessive ocular albinism. Hum Genet 1981; 56: 299-304.

4 Witkop CJ Jr. Inherited disorders of pigmentation. Clin Dermatol 1985; 3: 70-134

5 Christensen AM, Wallman J. Evidence that increase sclera growth under visual deprivation myopia in chicks. Invest Ophthalmol Vis Sci 1991; 32: 2143-50. 